\title{
Discriminating factors excluding patients from a catheter-based left atrial appendage closure and an outcome analysis of non-intervened and intervened patients
}

\author{
Christian Fastner, Claude Jabbour, Michael Behnes, Benjamin Sartorius, Annika Wenke, \\ Ibrahim El-Battrawy, Uzair Ansari, Martin Borggrefe, Ibrahim Akin
}

First Department of Medicine, University Medical Centre Mannheim (UMM), Faculty of Medicine Mannheim, University of Heidelberg, and DZHK (German Center for Cardiovascular Research) partner site Heidelberg/Mannheim, Mannheim, Germany

Submitted: 25 July 2019

Accepted: 7 January 2020

Arch Med Sci

DOI: https://doi.org/10.5114/aoms.2020.98652

Copyright (c) 2020 Termedia \& Banach

\section{Abstract}

Introduction: The catheter-based left atrial appendage closure (LAAC) has evolved as an alternative to oral anticoagulation (OAC) among non-valvular atrial fibrillation (AF) patients in whom long-term OAC is contraindicated. In daily practice, however, a sizeable number of patients who have been referred for an LAAC do not receive this intervention. This study aimed primarily to investigate the factors deterring the practice of an LAAC in referred AF patients, and secondarily to compare the complication rates of intervened patients with those who had refused the intervention within one year.

Material and methods: This retrospective single-centre study includes $200 \mathrm{pa}$ tients. After a thoroughly conducted clinical selection process, 161 of these patients (80.5\%) were excluded from receiving an LAAC intervention.

Results: An analysis comparing these patients to those receiving an LAAC reveales that a higher proportion of intervened patients had suffered a prior gastrointestinal bleeding (48.7 vs. $28.0 \% ; p=0.013$ ) as well as a haemorrhagic stroke ( 12.8 vs. $2.5 \% ; p=0.015)$, and was not anticoagulated the time of presentation (35.9 vs. $14.9 \% ; p=0.006$ ). The main reason for not conducting the procedure was patient refusal (62.1\%) followed by multimorbidity (16.8\%). The annual rate of ischaemic strokes and bleedings among patients refusing the intervention was $2.1 \%$ and $29.5 \%$, respectively, and this was not statistically different from the intervened patients (each $p>0.05$ ).

Conclusions: The reasons why patients did not undergo the catheter-based LAAC were mainly reluctance for the procedure and multimorbidity. Furthermore, it could be assumed that the potential benefit of the LAAC may not be realised within the first year.

Key words: atrial fibrillation, high bleeding risk, left atrial appendage, left atrial appendage closure, non-execution.

\section{Introduction}

Atrial fibrillation (AF) represents the most common cardiac arrhythmia with a current age-dependent prevalence of $1-2 \%$ in the western population [1]. The medium-term prognostic complications, with cerebral ischaemic stroke leading the way, make it a significant clinical disorder [2].

\author{
Corresponding author: \\ Christian Fastner MD \\ University Medical Centre \\ Mannheim \\ First Department of Medicine \\ Theodor-Kutzer-Ufer 1-3 \\ 68167 Mannheim, Germany \\ Phone: (+49) 6213832512 \\ Fax: (+49) 6213832012 \\ E-mail: \\ christian.fastner@umm.de
}


Many of the affected patients do not survive these complications, and a clear majority usually retain a permanent disability [3]. The $\mathrm{CHA}_{2} \mathrm{DS}_{2}$-VASc score has generally been applied to assess the patient's individual stroke risk, and used to tailor a risk-adjusted treatment strategy [3].

Oral anticoagulation (OAC) has evolved as the therapy of choice for stroke prevention in patients with AF [3]. However, in the real-life setting, a considerable proportion of these patients appear to be inadequately anticoagulated. This trend has been continually observed despite the introduction of the so-called direct oral anticoagulants (DOAC) [4-6]. Most of these patients either face a high bleeding risk as assessed by a HAS-BLED score $\geq 3$ points, or have been affected by major or recurrent bleeding $[3,7]$. Atrial fibrillation patients with concomitant dual antiplatelet therapy (DAPT), which is still needed for specific cases after percutaneous coronary intervention despite DOAC therapy, are prone to a relevantly increased bleeding risk $[8,9]$. Moreover, some patients face thromboembolic events despite effective OAC $[3,10]$. Such patients might benefit from a catheter-based left atrial appendage closure (LAAC) $[7,11,12]$.

Randomised controlled trials have proven the superiority of at least the WATCHMAN ${ }^{\text {TM }}$ device (Boston Scientific, Natick, MA, USA) compared to standard OAC with warfarin [13], and further studies suggested that OAC could be safely switched for antiplatelet agents after implantation, thus lowering the bleeding risk of the individual patient $[14,15]$.

Although the indications for catheter-based LAAC have been described in detail [16-18], there is a lack of data investigating the relevant number of patients referred for LAAC device implantation, and ending up not receiving it. Therefore, this study aimed to investigate the underlying reasons.

\section{Material and methods}

\section{Enrolment}

This analyses are based on a single-centre and all-comers study. All patients $\geq 18$ years of age referred for evaluation of LAAC device implantation between April 2014 and April 2015 were included. As this study aimed at evaluating reasons for the non-implantation of an LAAC device among all patients referred, specific exclusion criteria were not defined. The methods were carried out in accordance with the relevant local guidelines and regulations. All protocols were approved by the second medical ethics committee of the Faculty of Medicine Mannheim, University of Heidelberg, Germany (reference number 2014402M-MA- $\S 23 b$ MPG). Due to the retrospective character of this study, written informed consent was not obtained, but this was in line with the decision of the above-mentioned medical ethics committee.

\section{General clinical evaluation strategy}

In the course of the clinical management, all patients who are referred for LAAC to our cardiology centre undergo a three-step evaluation process. At any step, exclusion from the procedure is possible: Patients who are excluded at step 1 or 2 as well as patients who reject the intervention after the informative discussion are not earmarked for LAAC.

Step 1: Firstly, the medical history of every referred patient is analysed to verify consistency with existing European guideline recommendations, which suggest that LAAC may be considered in non-valvular AF patients with a high stroke risk and contraindications for long-term OAC [3]. High stroke risk is assumed in patients with all three types of AF when the $\mathrm{CHA}_{2} \mathrm{DS}_{2}$-VASc score is $\geq 2$ in men and $\geq 3$ in women [3]. Contraindications for long-term OAC are defined as follows (Table I): HAS-BLED score $\geq 3$ suggesting a high bleeding risk, relevant bleeding event with a tendency to recidivity, high risk of falls, e.g. in geriatric patients or patients with epilepsy, thromboembolic event despite effective OAC, and patient's refusal to take OAC after an in-depth consultation. DOAC prescription was taken into consideration in all eligible patients. As outlined in Table I, patients are also excluded at this step if contraindications for OAC withdrawal or catheter-based LAAC exist.

Step 2: Secondly, all matching patients are evaluated with respect to the potential benefit from the intervention by a multi-professional team including, where applicable, interventional cardiologists, geriatricians, neurologists, and oncologists. Multimorbid patients with limited life expectancy ( $<1$ year) and high interventional risk, e.g. compromised by a severely reduced left ventricular ejection fraction (LVEF $<30 \%$ ), are precluded. Ambiguous cases are discussed in the multi-professional team setting.

Step 3: Finally, all remaining patients are thoroughly informed about the procedure as well as the typical complications, and are asked for written informed consent for conducting the intervention.

\section{Retrospective processing of the data}

In a retrospective approach, we extracted the entire original evaluation data and the baseline characteristics from the medical documents for all patients referred to our centre during the time period mentioned above. By respecting 
Table I. Clinical three-step evaluation strategy

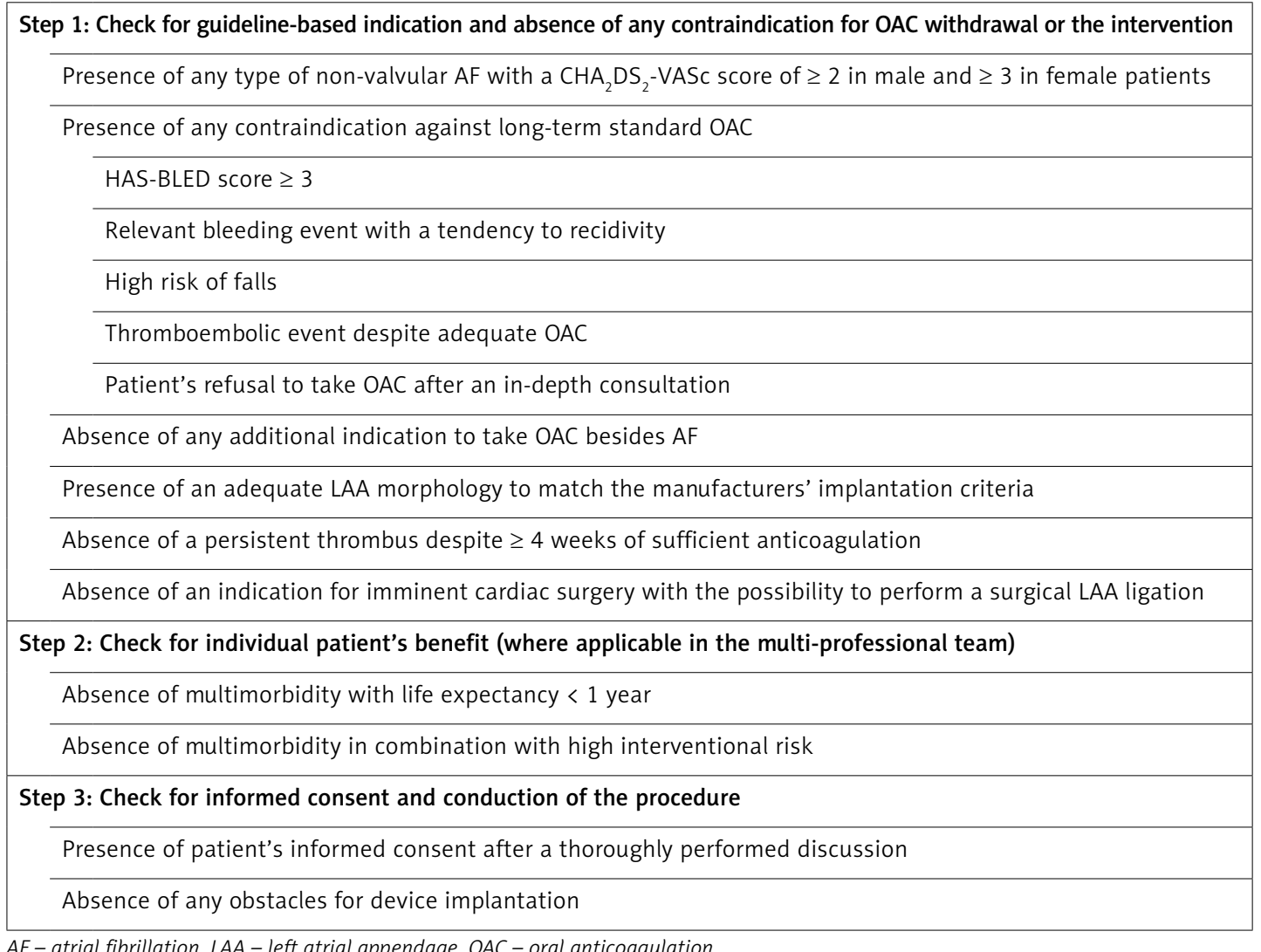

the three steps of the clinical evaluation strategy, all reasons contributing to exclusion were documented in an open approach.

In an additional step for those patients who had refused the intervention, we performed a retrospective one-year follow-up to investigate whether any complications related to the anticoagulation or the AF occurred after our recommendation. Moreover, one-year complication rates, including the procedural complications, were also documented in those patients who had received an LAAC. Intra-hospital para-device leaks $>5 \mathrm{~mm}$, pericardial effusions, overt bleedings, device embolisms, device thromboses, thromboembolisms, and cases of death were registered as procedural complications. All intervened patients received acetylsalicylic acid (ASA) 100 mg per day indefinitely and clopidogrel $75 \mathrm{mg}$ per day for half a year after the implantation.

\section{Outcome measures}

The primary outcome measure was the number of patients referred for $L A A C$ not receiving this intervention due to any underlying reason. A secondary outcome measure was the comparison of the complication rates among patients refusing the intervention to those of intervened patients during the retrospective one-year follow-up.

\section{Statistical analysis}

Statistical analyses were performed with SPSS Statistics version 24 (IBM, Armonk, NY, USA). Data are presented as medians with interquartile ranges $\left(25^{\text {th }}\right.$ and $75^{\text {th }}$ percentiles) or as total numbers with group-related percentages. Normal distribution of data was tested with the Kolmogorov-Smirnov test. In the case of normal distribution, the $t$-test was applied to compare scaled data. Scaled variables not normally distributed were compared using the Mann-Whitney $U$ test. Categorical variables were compared using the chi-squared test; in the case of low event rates the Fisher's exact test was used. The influence of the risk factor "triple therapy" on bleeding complications in the refusal group was investigated by a logistic regression analysis. These statistics were based on the available cases. $P$-values $<0.05$ (two-tailed) were considered significant.

\section{Results}

\section{Baseline characteristics}

Two hundred patients were included, and baseline characteristics were compared between those patients who underwent LAAC device implantation at the end of the clinical evaluation process 
Christian Fastner, Claude Jabbour, Michael Behnes, Benjamin Sartorius, Annika Wenke, Ibrahim El-Battrawy, Uzair Ansari, Martin Borggrefe, Ibrahim Akin

Table II. Baseline characteristics of the study collective

\begin{tabular}{|c|c|c|c|c|}
\hline Parameter & $\begin{array}{l}\text { All patients } \\
\quad(n=200)\end{array}$ & $\begin{array}{c}\text { Non-intervened } \\
\text { patients } \\
(n=161)\end{array}$ & $\begin{array}{l}\text { Intervened } \\
\text { patients } \\
(n=39)\end{array}$ & $P$-value* \\
\hline Male patients, $n(\%)$ & $131(65.5)$ & $105(65.2)$ & $26(66.7)$ & 0.864 \\
\hline Age [years] (IQR) & $77(71-81)$ & $77(70-81)$ & $79(73-82)$ & 0.123 \\
\hline $\mathrm{CHA}_{2} \mathrm{DS}_{2}$-VASc score (IQR) & $5(4-6)$ & $5(3-6)$ & $5(4-6)$ & 0.989 \\
\hline HAS-BLED score (IQR) & $4(3-5)$ & $4(3-5)$ & $4(3-5)$ & 0.394 \\
\hline \multicolumn{5}{|l|}{ Prior bleeding, each, $n$ (\%) } \\
\hline Intracranial & $18(9.0)$ & $12(7.5)$ & $6(15.4)$ & 0.121 \\
\hline Gastrointestinal & $64(32.0)$ & $45(28.0)$ & $19(48.7)$ & 0.013 \\
\hline Other & $73(36.5)$ & $64(39.8)$ & $9(23.1)$ & 0.052 \\
\hline \multicolumn{5}{|l|}{ LVEF, each, $n(\%)$} \\
\hline Normal (52-72) & $122(61.0)$ & $96(59.6)$ & $26(66.7)$ & 0.419 \\
\hline Borderline (41-51) & $28(14.0)$ & $19(11.8)$ & $9(23.1)$ & 0.069 \\
\hline Reduced (30-40) & $15(7.5)$ & $14(8.7)$ & $1(2.6)$ & 0.311 \\
\hline Severely reduced $(<30)$ & $35(17.5)$ & 32 (19.9) & $3(7.7)$ & 0.099 \\
\hline Arterial hypertension, $n$ (\%) & $197(98.5)$ & $158(98.1)$ & $39(100.0)$ & 1.000 \\
\hline Diabetes mellitus, $n(\%)$ & $64(32.0)$ & $55(34.2)$ & $9(23.1)$ & 0.245 \\
\hline \multicolumn{5}{|l|}{ Stroke, each, $n(\%)$} \\
\hline Ischaemic & $30(15.0)$ & $24(14.9)$ & $6(15.4)$ & 0.940 \\
\hline Haemorrhagic & $9(4.5)$ & $4(2.5)$ & $5(12.8)$ & 0.015 \\
\hline Both entities & $1(0.5)$ & $0(0)$ & $1(2.6)$ & - \\
\hline \multicolumn{5}{|l|}{ Coronary artery disease, each, $n(\%)$} \\
\hline Without prior myocardial infarction & $88(44.0)$ & $71(44.1)$ & $17(43.6)$ & 0.954 \\
\hline With prior myocardial infarction & $42(21.0)$ & $33(20.5)$ & $9(23.1)$ & 0.892 \\
\hline Peripheral arterial occlusive disease, $n$ (\%) & $76(38.0)$ & $65(40.4)$ & $11(28.2)$ & 0.222 \\
\hline Impaired renal function, $n(\%)$ & $135(67.5)$ & $111(68.9)$ & $24(61.5)$ & 0.487 \\
\hline \multicolumn{5}{|l|}{ OAC at time of presentation, each, $n(\%)$} \\
\hline Vitamin-K antagonist & $67(33.5)$ & $57(35.4)$ & $10(25.6)$ & 0.332 \\
\hline Dabigatran & $20(10.0)$ & $17(10.6)$ & $3(7.7)$ & 0.770 \\
\hline Rivaroxaban & $34(17.0)$ & $28(17.4)$ & $6(15.4)$ & 0.951 \\
\hline Apixaban & $30(15.0)$ & $28(17.4)$ & $2(5.1)$ & 0.077 \\
\hline Low-molecular-weight heparin & $11(5.5)$ & $7(4.3)$ & $4(10.3)$ & 0.230 \\
\hline None & $38(19.0)$ & $24(14.9)$ & $14(35.9)$ & 0.006 \\
\hline
\end{tabular}

Data are shown as numbers and percentages of patients, or as medians with interquartile range (IQR). * $P$-values for the comparison of both groups, $p<0.05$ indicates significant difference. LVEF - left ventricular ejection fraction, OAC - oral anticoagulation.

and those who did not. The baseline characteristics were mostly evenly distributed between both groups (Table II); in particular, $\mathrm{CHA}_{2} \mathrm{DS}_{2}$-VASC and HAS-BLED score did not significantly differ ( $p=0.989$ and $p=0.394$, respectively). The vast majority of patients had suffered from prior bleeding events $(77.5 \% ; p=0.135)$, whereby gastrointestinal (GI) bleeding events were more frequent among intervened patients (48.7 vs. $28.0 \%$; $p=0.013)$. While significantly more patients in the device group had experienced haemorrhagic strokes $(p=0.015)$, the percentage of overall intra- 
Table III. Reasons for non-execution in consideration of the three-step clinical evaluation strategy

\begin{tabular}{|c|c|}
\hline Reason for non-execution & $\begin{array}{c}\text { Non-intervened } \\
\text { patients } \\
(n=161)\end{array}$ \\
\hline \multicolumn{2}{|l|}{ Clinical evaluation strategy step 1} \\
\hline Patient without AF, $n(\%)$ & $8(5.0)$ \\
\hline No relevant contraindication for long-term standard $\mathrm{OAC}^{\star}, n(\%)$ & $11(6.8)$ \\
\hline Additional indication for OAC besides AF, $n(\%)$ & $5(3.1)$ \\
\hline LAA not matching implantation criteria, $n(\%)$ & $3(1.9)$ \\
\hline Persistent LAA thrombus, $n(\%)$ & $4(2.5)$ \\
\hline Patient with planned cardiac surgery and, therefore, indication for surgical LAA occlusion, $n(\%)$ & $1(0.6)$ \\
\hline \multicolumn{2}{|l|}{ Clinical evaluation strategy step 2} \\
\hline Multimorbidity with reduced life expectancy, $n(\%)$ & $27(16.8)$ \\
\hline \multicolumn{2}{|l|}{ Clinical evaluation strategy step 3} \\
\hline Refused consent for the intervention, $n(\%)$ & $100(62.1)$ \\
\hline Failed implantation procedure, $n(\%)$ & $2(1.2)$ \\
\hline
\end{tabular}

cranial bleeding events did not significantly differ (15.4 vs. $7.5 \% ; p=0.121)$. An appreciably higher percentage of patients in the device group had not been anticoagulated prior to the intervention (35.9 vs. $14.9 \%, p=0.006$ )

\section{Primary outcome measure}

One hundred and sixty-one (80.5\%) of all patients who were referred for LAAC met the primary outcome measure, meaning that they were excluded from receiving this intervention (Table III). Although most patients passed step 1 and 2 of the clinical evaluation strategy (70.5\%), 100 refused consent for the intervention, which was the main reason for exclusion from the intervention $(62.1 \%)$. Ten of these patients (10.0\%) were not anticoagulated at the time of presentation, whereby $3(3.0 \%)$ had a documented history of ischaemic stroke. Forty-nine of the refusing patients (49.0\%) had the indication due to a HAS-BLED score $\geq 3$, but had not suffered from a prior bleeding event within the last 6 months. Twenty-eight of these 49 patients $(57.1 \%)$ were on DOAC at the time of presentation.

A total of 24 patients $(12.0 \%$ of all referred patients) had to be excluded after the review of the medical records; almost half of these (5.5\%) presented without a relevant contraindication against long-term OAC. Twelve of 27 patients who were evaluated as multimorbid (44.4\%) had a severely reduced LVEF. In 2 patients the device implantation failed due to a tube-shaped atypical left atrial appendage (LAA) morphology.

\section{Secondary outcome measure}

In 95 patients who had refused the intervention (95.0\%) and 37 patients with a catheter-based closure of the LAA (94.9\%) one-year follow-up could be completed. Meanwhile, 1 patient received an LAAC elsewhere.

In the refusal group, 28 patients (29.5\%) suffered from a bleeding event including 8 cases (8.4\%) which fulfilled the International Society on Thrombosis and Haemostasis (ISTH) definition for major bleeding [19] (Table IV). Most bleeding events categorised as "other" were associated with a surgical procedure (53.3\%). In $87.5 \%$ of these cases, the OAC was paused longer than initially planned, but the exact period of interruption was not extractable from the existing data. $14.3 \%$ of patients with a bleeding event were on triple therapy with phenprocoumon or dabigatran, ASA 100 mg per day, and clopidogrel 75 mg per day after coronary stent implantation. Inversely, 5 out of 10 patients who received triple therapy suffered from a bleeding event during follow-up. However, in a logistic regression analysis, triple therapy was not significantly associated with an increased rate of bleedings (odds ratio: 2.696; 95\% confidence interval: $0.714-10.180 ; p=0.144)$. All patients who suffered from any thromboembolic event were on OAC. One patient with an ischaemic stroke who was known for phenprocoumon non-compliance was prescribed apixaban at the time of the event.

Twenty-three intervened patients (59.0\%) received a WATCHMAN ${ }^{T M}$ device, while the remaining 16 patients (41.0) received an AMPLATZER ${ }^{T M}$ 
Christian Fastner, Claude Jabbour, Michael Behnes, Benjamin Sartorius, Annika Wenke, Ibrahim El-Battrawy, Uzair Ansari, Martin Borggrefe, Ibrahim Akin

Table IV. Complications and adherence to medication during first year after refusal or implantation of an LAAC device

\begin{tabular}{|c|c|c|c|}
\hline & $\begin{array}{l}\text { Patients who refused } \\
\text { informed consent } \\
(n=95)\end{array}$ & $\begin{array}{l}\text { Intervened } \\
\text { patients } \\
(n=37)\end{array}$ & $P$-value* \\
\hline \multicolumn{4}{|l|}{ Efficacy and safety events } \\
\hline Transient ischaemic attack, $n(\%)$ & $0(0.0)$ & $0(0.0)$ & - \\
\hline Ischaemic stroke, $n(\%)$ & $2(2.1)$ & $0(0.0)$ & 1.000 \\
\hline Thromboembolic limb ischaemia, $n(\%)$ & $1(1.1)$ & $0(0.0)$ & 1.000 \\
\hline Thromboembolic mesenteric ischaemia, $n(\%)$ & $0(0)$ & $0(0.0)$ & - \\
\hline Cardiovascular death, $n(\%)$ & $4(4.2)$ & $1(2.7)$ & 1.000 \\
\hline Bleeding event, each $n(\%)$ & $28(29.5)$ & $10(27.0)$ & 0.948 \\
\hline Intracranial & $1(1.1)$ & $1(2.7)$ & 0.484 \\
\hline Gastrointestinal & $2(2.1)$ & $6(16.2)$ & 0.006 \\
\hline Epistaxis & $3(3.2)$ & $0(0.0)$ & 0.559 \\
\hline Skin & $3(3.2)$ & $0(0.0)$ & 0.559 \\
\hline Other & $15(15.8)$ & $3(8.1)$ & 0.397 \\
\hline Combined & $4(4.2)$ & $0(0.0)$ & 0.576 \\
\hline Major bleeding (ISTH definition) & $8(8.4)$ & 7 (18.9) & 0.321 \\
\hline Transfusion, $n(\%)$ & $9(9.5)$ & $6(16.2)$ & 0.429 \\
\hline \multicolumn{4}{|l|}{ Adherence to medication } \\
\hline No OAC prescribed, $n(\%)$ & $6(6.3)$ & - & - \\
\hline Incompliance with OAC, $n$ (\%) & $4(4.2)$ & - & - \\
\hline Need to interrupt OAC, $n(\%)$ & $19(20.0)$ & - & - \\
\hline
\end{tabular}

Data are shown as numbers and percentages of patients. ${ }^{*} P$-values for the comparison of both groups, $p<0.05$ indicates significant difference. ISTH - International Society on Thrombosis and Haemostasis, OAC - oral anticoagulation.

Amulet $^{\top M}$ (St. Jude Medical, St. Paul, MN, USA). During hospital stay, 1 patient revealed bleeding from the access site and was in need of a blood transfusion. Another patient suffered from a pericardial effusion, which could be treated conservatively. One WATCHMAN ${ }^{\mathrm{TM}}$ device dislodged intraprocedurally. It was snared, and a new WATCHMAN ${ }^{\top M}$ device was safely placed thereafter. An AMPLATZER ${ }^{T M}$ Amulet $^{T M}$ was found in the abdominal aorta during a routine follow-up visit half a year after the implantation. The device was retrieved interventionally, and the patient was switched back to DOAC at his request. He faced no further complication during follow-up. No para-device leak > $5 \mathrm{~mm}$, device thrombosis, intrahospital thromboembolism, or procedure-related death was evident. Within one year complication rates did not significantly differ between the two patient groups except for a significantly higher rate of $\mathrm{Gl}$ bleedings among the intervened patients (16.2 vs. $2.1 \%, p=0.006$; Table IV) out of which $80.0 \%$ occurred in the initial DAPT period. While both GI bleeding events in the refusal group were associated with multiple blood transfusions, none of those in the device group required a transfusion. No bleeding related to a surgical procedure was registered in the intervened group.

\section{Discussion}

Despite all the advantages, the LAAC intervention is associated with specific procedural risks [18]. Therefore, careful patient selection is indispensable. In our collective, $80.5 \%$ of patients referred for LAAC were not intervened, and, therefore, were deselected via a clinical three-step evaluation strategy, which was developed in our centre to manage this demand. Less than one fifth of all assigned patients (12.0\%) had to be excluded in step 1 as they did not meet the guideline requirements, which demonstrated an adequate allocation practice. It is crucial to identify patients with an additional indication for OAC besides AF in this step. For this reason, 5 patients $(2.5 \%)$ with recurrent pulmonary embolism were exclud- 
ed [20]. During pre-interventional transoesophageal echocardiography, 3 patients (1.5\%) revealed an LAA anatomy which did not match the manufacturers' recommendations on landing zone specifications [21].

For the WATCHMAN ${ }^{\text {TM }}$ device, superiority for allcause mortality could be demonstrated only after 3.8 years of follow-up [13]. Therefore, the individual patient's life expectancy should exceed this period, which was addressed by step 2 of the clinical evaluation process. $16.8 \%$ of exclusions were due to multimorbidity or another cause of limited life expectancy. Among multimorbid patients, a severely reduced LVEF may lead to an unacceptably high interventional risk [22, 23], especially in the context of deep conscious sedation, which is needed during the procedure [24]. Almost one half of the patients excluded for multimorbidity (44.4\%) had a severely reduced LVEF.

The remaining 141 patients who revealed both a guideline-based indication for thromboembolic prophylaxis and a contraindication for long-term OAC as well as the principle eligibility for this cardiac intervention were thoroughly informed about the procedure and the specific risks. Thereafter, 100 patients $(50.0 \%)$ refused consent for the intervention. The main subjective reason for refusing the intervention despite a high bleeding risk under OAC might have been the fact that almost half of these patients (49.0\%) had not experienced a bleeding event within the past 6 months. Therefore, they might have had a subjective feeling of safety under OAC.

It was interesting to note that a considerable number of patients (29.5\%) were evaluated as ineligible for long-term OAC, but had refused the intervention suffered from a bleeding event during follow-up, including about $8.4 \%$ with a major bleeding event. Concordant with these figures, the expected annual major bleeding rate in patients with a HAS-BLED score of 4 is $8.7 \%$ [25]. All these patients were on anticoagulation therapy except for 1 patient who died several days after the LAAC recommendation due to a second intracerebral bleeding event while the anticoagulation therapy was paused. In contrast to other published data [8, 9], an additional triple therapy did not significantly influence the rate of bleeding complications. Numerous bleeding events were associated with surgical procedures (28.6\%). It is hence conceivable that patients awaiting extended or repetitive surgical procedures are potential beneficiaries of an LAAC.

Within one year of follow-up, overall and major bleeding rates were not yet significantly different between the groups in our limited sample. While the Gl bleeding rate was significantly higher among the intervened patients, none of these patients was in need of a blood transfusion. In contrast, both patients with GI bleedings who had refused the LAAC received multiple transfusions. Moreover, the intervened patients had been affected significantly more often by GI bleedings prior to the intervention, and the baseline characteristics imply that they were prone to a higher bleeding risk as expressed by a significantly higher percentage of patients with prior haemorrhagic strokes and patients who were not anticoagulated. These items are not adequately addressed by the HAS-BLED score, which revealed no statistically significant difference.

Addressing issues concerning the timing of complications, a large randomised clinical trial revealed that most bleeding events associated with an LAAC occurred in the early period after implantation [13]. During this period, our intervened patients received a DAPT, which was shown to cause at least the same magnitude of bleeding events as an OAC in AF patients [26]. Therefore, the superiority of the LAAC procedure can be expected only in the long run [13]. However, the number of periprocedural complications was low in our collective as well as comparable to other published data [18], and no patient revealed permanent disability or died due to any of these intrahospital complications. Limited by a relatively small sample size, no statistically significant difference was revealed in rates of thromboembolic complications during one-year follow-up either.

An inherent limitation of this retrospective observational study is that we only included patients who were referred for LAAC evaluation by their treating physician. There might be a certain number of AF patients who are prone to a high bleeding risk under OAC, or who are not anticoagulated, but are not recommended for LAAC evaluation. A subsequent study should, therefore, focus on this issue. Information about the patients' height and weight could not be extracted from the original data to a relevant extent. Furthermore, the follow-up analyses were based on a very limited sample size of intervened patients as numerous patients from our sample refused the intervention. As in other retrospective analyses, the total sample size could not be calculated to reveal a statistically significant difference for a secondary outcome measure. Therefore, the results of the follow-up analyses have to be interpreted with caution. The fact that no statistically significant difference was present in several items might be due to the low event rates in the small sample. However, despite all the limitations of this observational registry, it definitely serves as a data source for an unstudied topic.

In conclusion, this retrospective study showed that a relevant proportion of referred patients 
were deselected during the clinical evaluation process. While preselection of patients according to guideline recommendations appeared adequate, various reasons for exclusion existed, where the patient's refusal was the main issue. The refusal of the interventional approach might be based on a subjective feeling of safety under OAC. However, a substantial percentage of patients who had refused the intervention experienced bleeding events during follow-up.

\section{Conflict of interest}

The authors declare no conflict of interest.

\section{References}

1. Go AS, Hylek EM, Phillips KA, et al. Prevalence of diagnosed atrial fibrillation in adults: national implications for rhythm management and stroke prevention: the AnTicoagulation and Risk Factors in Atrial Fibrillation (ATRIA) Study. JAMA 2001; 285: 2370-5.

2. Gage BF, Waterman AD, Shannon W, Boechler M, Rich MW, Radford MJ. Validation of clinical classification schemes for predicting stroke: results from the National Registry of Atrial Fibrillation. JAMA 2001; 285: 2864-70.

3. Kirchhof P, Benussi S, Kotecha D, et al. 2016 ESC Guidelines for the management of atrial fibrillation developed in collaboration with EACTS. Eur Heart J 2016; 37: 2893 962.

4. Rose AJ, Goldberg R, McManus DD, et al. Anticoagulant prescribing for non-valvular atrial fibrillation in the veterans health administration. J Am Heart Assoc 2019; 8: e012646.

5. Alamneh EA, Chalmers L, Bereznicki LR. Suboptimal use of oral anticoagulants in atrial fibrillation: has the introduction of direct oral anticoagulants improved prescribing practices? Am J Cardiovasc Drugs 2016; 16: 183-200.

6. Labuz-Roszak B, Machowska-Majchrzak A, Skrzypek M, et al. Antiplatelet and anticoagulant therapy in elderly people with type 2 diabetes mellitus in Poland (based on the PolSenior Study). Arch Med Sci 2017; 13: 1018-24.

7. Peritz DC, Chung EH. Left atrial appendage closure: an emerging option in atrial fibrillation when oral anticoagulants are not tolerated. Cleve Clin J Med 2015; 82: 167-76.

8. Barbieri L, Verdoia M, Schaffer A, Suryapranata H, De Luca G. Risk and benefits of triple therapy in patients undergoing coronary stent implantation requiring oral anticoagulation: a meta-analysis of 16 studies. Cardiovasc Drugs Ther 2016; 30: 611-22.

9. Haller PM, Sulzgruber P, Kaufmann C, et al. Bleeding and ischaemic outcomes in patients treated with dual or triple antithrombotic therapy: systematic review and meta-analysis. Eur Heart J Cardiovasc Pharmacother 2019; 5: 226-36.

10. Wankowicz P, Nowacki P, Golab-Janowska M. Risk factors for ischemic stroke in patients with non-valvular atrial fibrillation and therapeutic international normalized ratio range. Arch Med Sci 2019; 15: 1217-22.

11. Fastner C, Hoffmann L, Aboukoura M, et al. Real-world experience comparing two common left atrial appendage closure devices. BMC Cardiovasc Disord 2018; 18: 171.
12. Fastner C, Behnes M, Henzler T, Borggrefe M, Akin I. Lack of silent cerebral ischemic events: a case series of patients after left atrial appendage closure. Clin Res Cardiol 2018; 107: 91-3.

13. Reddy VY, Sievert H, Halperin J, et al. Percutaneous left atrial appendage closure vs warfarin for atrial fibrillation: a randomized clinical trial. JAMA 2014; 312: 198898.

14. Reddy VY, Mobius-Winkler S, Miller MA, et al. Left atrial appendage closure with the Watchman device in patients with a contraindication for oral anticoagulation: the ASAP study (ASA Plavix Feasibility Study With Watchman Left Atrial Appendage Closure Technology). J Am Coll Cardiol 2013; 61: 2551-6.

15. Urena M, Rodes-Cabau J, Freixa X, et al. Percutaneous left atrial appendage closure with the AMPLATZER cardiac plug device in patients with nonvalvular atrial fibrillation and contraindications to anticoagulation therapy. J Am Coll Cardiol 2013; 62: 96-102.

16. Pison L, Potpara TS, Chen J, et al. Left atrial appendage closure-indications, techniques, and outcomes: results of the European Heart Rhythm Association Survey. Europace 2015; 17: 642-6.

17. Fastner C, Behnes M, Sartorius B, et al. Left atrial appendage morphology, echocardiographic characterization, procedural data and in-hospital outcome of patients receiving left atrial appendage occlusion device implantation: a prospective observational study. BMC Cardiovasc Disord 2016; 16: 25.

18. Boersma LV, Schmidt B, Betts TR, et al. Implant success and safety of left atrial appendage closure with the WATCHMAN device: peri-procedural outcomes from the EWOLUTION registry. Eur Heart J 2016; 37: 2465-74.

19. Schulman S, Kearon C, Subcommittee on Control of Anticoagulation of the Scientific and Standardization Committee of the International Society on Thrombosis and Haemostasis. Definition of major bleeding in clinical investigations of antihemostatic medicinal products in non-surgical patients. J Thromb Haemost 2005; 3:692-4.

20. Konstantinides SV, Meyer G, Becattini C, et al.; ESC Scientific Document Group. 2019 ESC Guidelines for the diagnosis and management of acute pulmonary embolism developed in collaboration with the European Respiratory Society (ERS). Eur Heart J 2020; 41: 543-603.

21. Mobius-Winkler S, Majunke N, Sandri M, et al. Percutaneous left atrial appendage closure: technical aspects and prevention of periprocedural complications with the watchman device. World I Cardiol 2015; 7: 65-75.

22. Henriques JP, Ouweneel DM, Naidu SS, et al. Evaluating the learning curve in the prospective Randomized Clinical Trial of hemodynamic support with Impella 2.5 versus Intra-Aortic Balloon Pump in patients undergoing high-risk percutaneous coronary intervention: a prespecified subanalysis of the PROTECT II study. Am Heart J 2014; 167: 472-479.e5.

23. Al-Husami W, Yturralde F, Mohanty G, et al. Single-center experience with the TandemHeart percutaneous ventricular assist device to support patients undergoing high-risk percutaneous coronary intervention. J Invasive Cardiol 2008; 20: 319-22.

24. Sumler ML Andritsos MJ, Blank RS. Anesthetic management of the patient with dilated cardiomyopathy undergoing pulmonary resection surgery: a case-based discussion. Semin Cardiothorac Vasc Anesth 2013; 17: 9-27. 
25. Pisters R, Lane DA, Nieuwlaat R, de Vos CB, Crijns HJ, Lip GY. A novel user-friendly score (HAS-BLED) to assess 1 -year risk of major bleeding in patients with atrial fibrillation: the Euro Heart Survey. Chest 2010; 138: 1093-100.

26. Hohnloser SH, Pajitnev D, Pogue J, et al. Incidence of stroke in paroxysmal versus sustained atrial fibrillation in patients taking oral anticoagulation or combined antiplatelet therapy: an ACTIVE W Substudy. J Am Coll Cardiol 2007; 50: 2156-61. 